\title{
Detection of Brain Tumor using Hybrid Topology Preserving Map
}

\author{
M.C.Jobin Christ \\ Assistant Professor \\ Department of Biomedical Engineering, \\ Adhiyamaan College of Engineering, Hosur, \\ Tamilnadu, India
}

\author{
R.M.S.Parvathi \\ Phd, Principal, \\ Sengunthar College of Engineering, Tiruchencode, \\ Tamilnadu, India
}

\begin{abstract}
Segmentation of an image is the division or separation of the image into disjoint regions of similar feature. In clinical practice, Magnetic Resonance Imaging (MRI) is used to distinguish pathologic tissue from normal tissues, especially for the detection of brain tumors. Among the thousands of segmentation methods, clustering algorithm is one of the powerful tools in segmenting medical images. In this paper we proposed a new approach based on Hybrid Topology Preserving Map (HTPM) and Adaptive Mean Shift (AMS) algorithm which combines the advantages of both mean shift based clustering algorithm and Hierarchical Topology Preserving Map. And also the performance of the proposed method is compared with the traditional method Adaptive Mean Shift (AMS) algorithm.
\end{abstract}

Keywords: AMS, HTPM, MRI, Segmentation.

\section{INTRODUCTION}

Segmentation is an important process in biomedical image processing. For image registration, shape analysis, visualization and quantitative analysis, etc. one should do the segmentation process initially. Segmentation of an image is the division or separation of the image into disjoints regions of similar feature [1]. Magnetic Resonance (MRI) is used to discriminate pathologic tissue from normal tissue, especially for brain tumors. Three main regions of brain, White Matter (WM), Gray Matter (GM) and Cerebrospinal Fluid (CSF) are the important subject of study in brain imaging. Each year over 190,000 people in the United States and 10,000 people in Canada are diagnosed with various types of brain tumor. Brain tumors are the second leading cause of cancer based deaths among children in the age group between 0-19 years. And also they are the second leading cause of cancer based deaths in young men up to age 39 years and the fifth leading cause of cancer based deaths among young women between ages 20-39 years. There are over 120 different types of brain tumors including malignant and benign making effective treatment highly complicated [2]. The first step to minimize the death rate of brain tumor is developing effective diagnosing method. MRI is one of the best imaging system to diagnose brain tumor, even though it is crucial for the clinicians to diagnose the early stage of brain tumor. So image segmentation is the only possible tool for early detection of brain tumor. Manual segmentation by an expert will consume more time and it is very difficult to do proper segmentation. Hence automatic segmentation algorithms are preferred in diagnosing brain tumor. Instead of performing segmentation with a single algorithm, if multiple technologies joined together means we may get effective solution. In this paper we proposed a novel hybrid approach for the detection of brain tumor which is based on Adaptive Mean Shift algorithm and Hybrid Topology Preserving Map.

\section{LITERATURE SURVEY}

Image segmentation algorithms are generally classified into two types, supervised and unsupervised. Unsupervised algorithms are fully automatic and partition the regions in feature space with high density. The different unsupervised algorithms are Feature-Space Based Techniques, Clustering (K-means algorithm, C-means algorithm, E-means algorithm, etc), Histogram thresholding, Region Based Techniques (Split-and-merge techniques, Region growing techniques, Neural-network based techniques, Edge Detection Techniques, Fuzzy Techniques, etc $[1,3,4,5]$. T.Logeswari et al described about HSOM and Fuzzy C-means algorithm for the detection of brain tumor [6]. S. Murugavalli et al described about Neuro-fuzzy technique for implementation of brain tumor detection [7]. V.P.Gladis Pushpa Rathi et al described HSOM along with wavelet packets for detection and characterization of brain tumors [8]. In our previous paper, we described about the integration of clustering algorithms with marker controlled watershed transform for the segmentation of brain images [9]. Arnaldo Mayer and Hayit Greenspan described about adaptive mean shift algorithm for magnetic resonance brain image segmentation [10].

\section{OVERVIEW OF HYBRID METHODS}

More than one technology is joined together to solve a particular problem means that system is called as a hybrid system. The soft computing technologies like neural networks, fuzzy logic and genetic algorithms are integrated themselves and yielding efficient results by solving complex problems. Hybrid systems are divided into three groups. They are sequential hybrid systems, auxiliary hybrid systems and embedded hybrid systems [11]. Sequential hybrid systems use two technologies in a pipeline-like method. One technology's output will be the input of another technology's input. In auxiliary hybrid systems, one technology calls the other as a subroutine to process information needed by it. The second technology processes the information given by the first and gives back to the first technology. In embedded hybrid systems, both technologies are intertwined together. Here fusion between the technologies made such that no one can be used without the other for problem solving. This is the absolute method of hybridization. Figure. 1 shows a sequential hybrid system. 


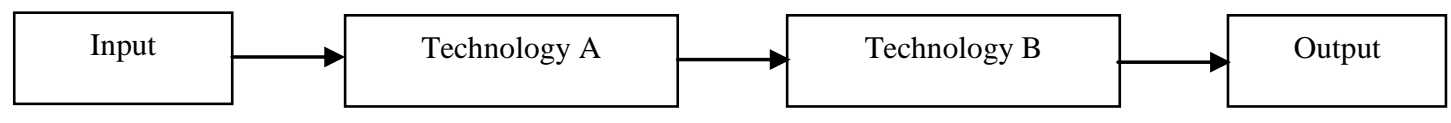

Figure. 1 A Sequential Hybrid System

\section{PROPOSED ALGORITHM}

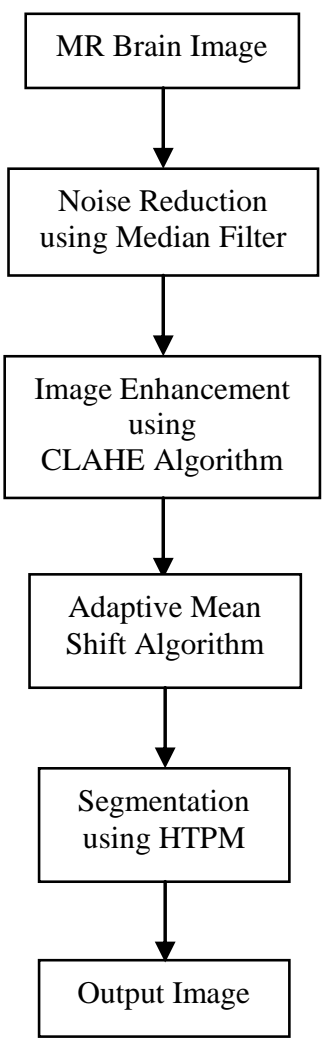

Figure. 2 Flow Diagram of Proposed Algorithm

Figure. 2 describes the proposed algorithm. MR brain image obtained from patient is stored in MATLab. Noise presented in the image is removed by median filtering. After removing the noise using median filter the image is enhanced using Contrast -Limited Adaptive Histogram Equalization (CLAHE) method. CLAHE operates on small regions in the image, called tiles, rather than the entire image. Each tile's contrast is enhanced. Then the enhanced image is segmented using An Adaptive Mean Shift Algorithm and Hybrid Topology Preserving Map. An adaptive mean-shift methodology is utilized in order to classify brain voxels into one of three main tissue types: Gray matter, White matter, and Cerebro-spinal fluid. The MRI image space is represented by a high-dimensional feature space that includes multimodal intensity features as well as spatial features. An adaptive mean-shift algorithm clusters the joint spatial-intensity feature space, thus extracting a representative set of high-density points within the feature space, otherwise known as modes. Tissue segmentation is obtained by a follow-up phase of intensity-based mode clustering into the three tissue categories. By its nonparametric nature, adaptive mean-shift can deal successfully with non-convex clusters and produce convergence modes that are better candidates for intensity based classification than the initial voxels. The output of AMS algorithm is then given to the Topology Preserving Map for the clear identification of the tissues. The method for placing a vector from data space onto the map is to find the node with the closest weight vector to the vector taken from data space and to assign the map coordinates of this node to our vector. Euclidean to all weight vectors is computed. The neuron with weight vector most similar to the input is called the best matching unit (BMU). The weights of the BMU and neurons close to it in the TPM lattice are adjusted towards the input vector. The magnitude of the change decreases with time and with distance from the BMU. Steps of updating weights in HTPM are following.

1. Calculate the winning neuron using

$$
\|\mathrm{x}-\mathrm{wc}\|=\operatorname{maxi}\{[=\mathrm{x}-\mathrm{wi}]\}
$$

Where, $\mathrm{x}$ is a neuron, wi is the weight vector.

2. Calculate the cluster centers

$$
\mathrm{C}=(\mathrm{N} / 2) 1 / 2
$$

3. Compute the Euclidean distances

$$
\mathrm{Dij}=\mathrm{CCp}-\mathrm{Cn}
$$

4. Update the partition matrix

$$
U_{i j}=\frac{1}{\sum_{k=l}^{c}\left(\frac{d_{i j}}{d_{i j}}\right)^{2 /(m-l)}}
$$

5. Repeat step 5 until $\operatorname{Max}\left[\left|\mathrm{U}_{\mathrm{ij}}(\mathrm{k}+1)-\mathrm{U}_{\mathrm{ij}}(\mathrm{k})\right|\right]<\mathrm{T}$ is satisfied.

6. Calculate the average clustering points

$$
C_{i}=\sum_{i=1}^{c} J_{i}=\sum_{i=1}^{c} \sum_{j=1}^{n} U_{i j}{ }^{n} d_{i j}^{2}
$$

7. Compute the adaptive threshold

Adaptive threshold $=\max$ (Adaptive threshold, ci) $\mathrm{i}=1$...n In the first step, the algorithm selects the initial cluster from HTPM Clustering algorithm.

\section{RESULTS AND ANALYSIS}

The following figures show the outputs of various stages of our proposed algorithm.

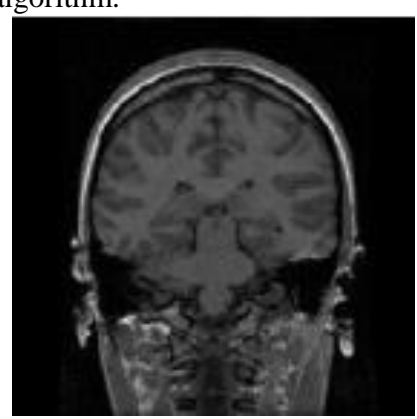

Figure. 3 Input Image 


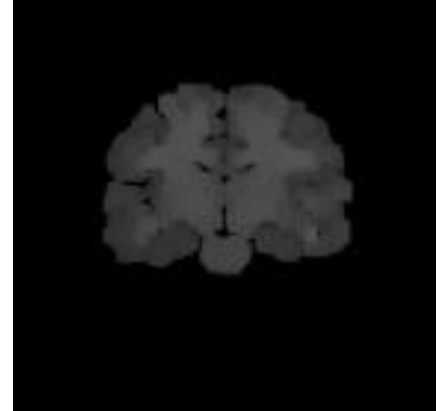

Figure. 4 Intensity Image

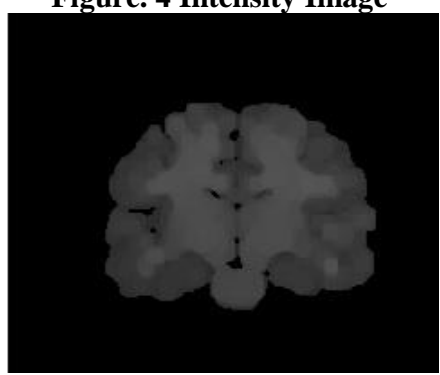

Figure. 5 Denoised Image

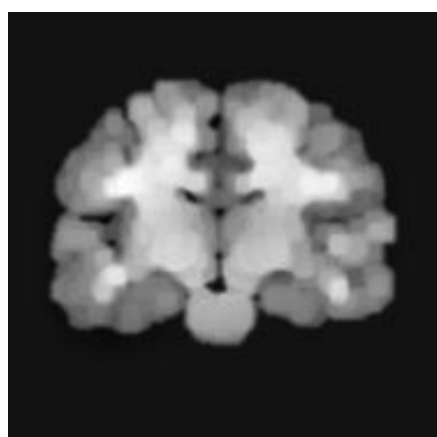

Figure. 6 High Intensity Image
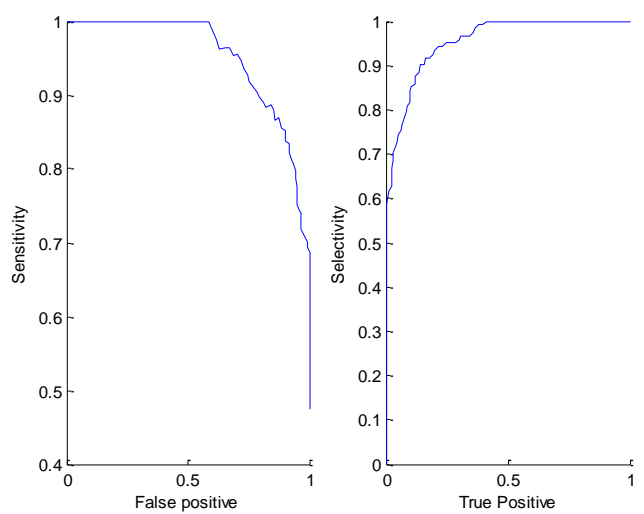

Figure. 7 Sensitivity and Selectivity graph
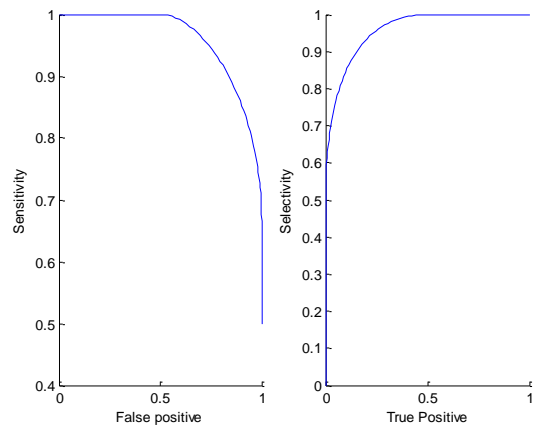

Figure. 8 Sensitivity and Selectivity of Output

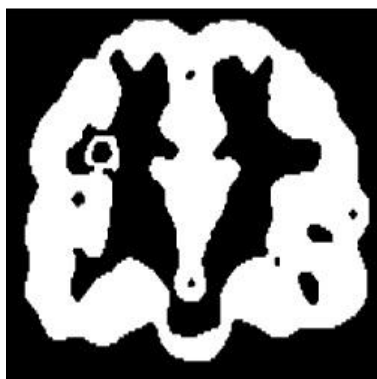

Figure. 9 Segmented Image using HTPM

The MRI brain image is given as the input. Here the coronal slice of brain is taken as input. The input image is then shown by means of different intensities by performing morphological operation to highlight the foreground and background pixels. Noise present in the image is then removed by using median filter. Noise is removed by Median filter. The high intensity region is obtained after the removal of noise. The contrast of the image is improved by applying Contrast Limited Adaptive Histogram Equalization algorithm. The sensitivity and the selectivity for the AMS algorithm are 0.9776 and 1 respectively. The image obtained after applying AMS is then used for fusing with Hybrid Topology Preserving Map. The sensitivity and the selectivity for the HTPM algorithm are 0.9800 and 1 .

\section{CONCLUSION}

In this paper, Hybrid Topology Preserving Map with Adaptive Mean Shift algorithm is described to achieve segmentation of brain MR images. From the AMS algorithm, high intensity image is segmented. Then HTPM is applied over high intensity image. The sensitivity values showed that HTPM provided better sensitivity than high intensity image. So this will give a better understanding of hybrid technologies in the field of medical image segmentation. 


\section{REFERENCES}

[1] H.S.Prasantha et. al, 2010, "Medical Image Segmentation", International Journal on Computer Science and Engineering, Vol. 02, No. 04.

[2] National Brain Tumor Society, www.braintumor.org.

[3] B.Chanda, D.Dutta Majumder, 2008Digital Image Processing and Analysis, Prentice Hall of India Pvt. Ltd.

[4] Rafael C. Gonzalez, Richard E. Woods, Digital Image processing, $2^{\text {nd }}$ edition, Pearson Education, 2007, pp.589656.

[5] S.Jayaraman, et.al, 2009Digital Image Processing, Tata McGraw Hill Education Pvt. Ltd.

[6] T.Logeswari and M.Karnan, 2010, "An Improved Implementation of Brain Tumor Detection Using Segmentation Based on Hierarchical Self Organizing Map", International Journal of Computer Theory and Engineering, Vol. 2, No. 4, pp: 591-595.

[7] S. Murugavalli and V. Rajamani, 2007, "An Improved Implementation of Brain Tumor Detection Using
Segmentation Based on Neuro Fuzzy Technique", Journal of Computer Science, Vol. 3, No. 11, pp: 841846

[8] V.P.Gladis Pushpa Rathi and Dr.S.Palani, 2011, "Detection and Characterization of Brain Tumor Using Segmentation based on HSOM, Wavelet packet feature spaces and ANN", IEEE, pp: 274-277.

[9] M.C.Jobin Christ and R.M.S.Parvathi, 2011, "Segmentation of Medical Image using Clustering and Watershed Algorithms", American Journal of Applied Sciences, Vol. 8, No. 12, pp: 1349-1352.

[10] Arnaldo Mayer and Hayit Greenspan, 2009, "An Adaptive Mean-Shift Framework for MRI Brain Segmentation", IEEE transactions on medical imaging, Vol. 28, No. 8, pp: 1238-1250.

[11] S.Rajasekaran and G.A. Vijayalakshmi Pai, 2011, "Neural Networks, Fuzzy Logic, and Genetic Algorithms Synthesis and Applications", PHI Learning Private Ltd., pp : 297-300. 